\title{
SUMS, PRODUCTS AND CONTINUITY OF BOREL MAPS IN NONSEPARABLE METRIC SPACES
}

\author{
R. W. HANSELL
}

(Communicated by R. Daniel Mauldin)

\begin{abstract}
We give a comparatively simple proof that the complex map $(f, g): T \rightarrow X \times Y$ will be (Borel measurable) of class $\alpha+\alpha$, whenever $f$ and $g$ are of class $\alpha$, for not necessarily separable metric spaces $T, X$ and $Y$. The Borel measurability of other types of maps, such as the sum of two vectorvalued maps, is easily deduced from this. A general version of this result, applicable to abstract measurable spaces, is also proven.

Our second principal result shows that, if $f: T \times X \rightarrow Y$ is continuous in each variable separately, where $X$ and $Y$ are metrizable, then $f$ has a $\sigma$-locally finite function base of closed sets, and thus will be continuous at each point of the complement of some first category subset of $T \times X$.
\end{abstract}

1. Borel measurability of complex maps. In this section we are primarily concerned with the following question: If $f: T \rightarrow X$ and $g: T \rightarrow Y$ are Borel (measurable) maps, for metric spaces $T, X$ and $Y$, is the "complex map" $(f, g)$, which takes $t$ to the pair $(f(t), g(t))$, also a Borel map? When $X$ and $Y$ are separable it is relatively easy to show that $(f, g)$ will be, in fact, of class $\alpha$ whenever $f$ and $g$ are of class $\alpha[\mathbf{K 1}, \S 31, \mathrm{VI}]$. (Actually, only one of $X$ or $Y$ need be separable for this to hold, as is shown in Theorem 1 below.)

The Borel measurability of $(f, g)$ is of fundamental importance since the Borel measurability of other functions can often be easily deduced from this. For example, if $X=Y$ is a Banach space, then the vector sum $f+g$ can be expressed as the composition of $(f, g)$ followed by the continuous map $+: X \times X \rightarrow X$; hence $f+g$ will be a Borel map whenever $(f, g)$ is. (The reader is referred to $[\mathbf{S}]$ for a more detailed discussion of this.) The Borel measurability of cartesian products of maps can similarly be analyzed in terms of certain complex maps (see, e.g., [K1, 331 , $\mathrm{VI}]$ ).

When $X$ and $Y$ are nonseparable metric spaces, the problem of whether or not the complex map $(f, g)$ is Borel measurable had remained open for some time, and was first raised by Kuratowski in 1935 [K2, §8] (see also the seminal paper of D. Montgomery $[\mathbf{M}]$ concerning this and related problems on Borel maps in nonseparable metric spaces). Although little was known of the structure of nonseparable metric spaces at that time, a complete answer to this problem was not given until 1983 , when it was shown that $(f, g)$ must be of class $\alpha+\alpha$ whenever $f$ and $g$ are of class $\alpha$; and that this class could not be lowered, even in the case when $\alpha=1$, given the existence of certain models of set theory (see $[\mathbf{H 2}, \S 6]$ ). The proof of this,

Received by the editors September 14, 1987.

1980 Mathematics Subject Classification (1985 Revision). Primary 28A05, 54H05.

Key words and phrases. Borel measurable maps, nonseparable metric spaces, complex maps, separately continuous maps. 
however, required a number of technical preliminary results dealing with the concept of a "hereditarily additive family" and a process of taking successive iterations of "descriptive operations."

Here we will give a surprisingly simple proof of this result, using only rudimentary properties of Borel sets in metric spaces, and which has the additional merit of showing in a more natural way how the class $\alpha+\alpha$ arises.

THEOREM 1. Let $f: T \rightarrow X$ and $g: T \rightarrow Y$ be Borel maps of class $\alpha$ (for some countable ordinal $\alpha$ ), for metric spaces $T, X$ and $Y$. Then the complex map $(f, g): T \rightarrow X \times Y$ is a Borel map of class $\alpha+\alpha$. If one of $X$ or $Y$ is separable, then $(f, g)$ will be of class $\alpha$.

ProOF. Suppose $X$ is separable, and thus has a countable open base $\left\{G_{n}: n \in\right.$ $\omega\}$. Given an open set $W \subset X \times Y$, define

$$
V_{n}=\bigcup\left\{V: V \text { is open in } Y \text { and } G_{n} \times V \subset W\right\}
$$

for each $n \in \omega$. Then $W=\bigcup\left\{G_{n} \times V_{n}: n \in \omega\right\}$, and so

$$
(f, g)^{-1}(W)=\bigcup_{n \in \omega} f^{-1}\left(G_{n}\right) \cap g^{-1}\left(V_{n}\right)
$$

is a countable union of sets of additive class $\alpha$, hence again of this class. This proves that $(f, g)$ is of class $\alpha$. The result follows similarly for the case when $Y$ is separable.

For the general case, we will first prove that any complex map consisting of a continuous map and a Borel map of class $\alpha$ will be a Borel map of class $\alpha$. Thus, assuming the notation of the theorem, suppose $f$ is continuous and $g$ is of class $\alpha$. Let $\left\{G_{a n}: a \in A_{n}, n \in \omega\right\}$ be an open base for $X$ where each subcollection corresponding to a fixed $n$ is locally finite in $X$. Let $W \subset X \times Y$ be a given open set and define

$$
V_{a n}=\bigcup\left\{V: V \text { is open in } Y \text { and } G_{a n} \times V \subset W\right\}
$$

for each $a \in A$ and $n \in \omega$. Then $W=\bigcup\left\{G_{a n} \times V_{a n}: a \in A_{n}\right.$ and $\left.n \in \omega\right\}$, hence

$$
(f, g)^{-1}(W)=\bigcup_{n \in \omega} \bigcup_{a \in A_{n}} f^{-1}\left(G_{a n}\right) \cap g^{-1}\left(V_{a n}\right) \text {. }
$$

Since $f$ is continuous and the union of a locally finite collection of sets of additive class $\alpha$ is again of additive class $\alpha$ (see [E]), it follows from the above formula that $(f, g)^{-1}(W)$ is of additive class $\alpha$. This proves that $(f, g)$ is a Borel map of class $\alpha$.

Now suppose both $f$ and $g$ are of class $\alpha$, and let us show that the map $(f, g)$ is itself the composition of two complex maps each consisting of a continuous map and a Borel map of class $\alpha$. It will then follow from the above that $(f, g)$ is a composition of two maps of class $\alpha$, and hence of class $\alpha+\alpha[\mathbf{K 1}, \S 31, \mathrm{III}]$.

Let $\operatorname{Gr}(f)$ denote the graph of $f$ as a subspace of $T \times X$, let $f^{*}: \operatorname{Gr}(f) \rightarrow X$ denote the map $(t, f(t)) \mapsto f(t)$, let $g^{*}: \operatorname{Gr}(f) \rightarrow Y$ be the composition of the continuous map $(t, f(t)) \mapsto t$ followed by the map $g$, and let $1_{T}$ denote the identity map for $T$. Then $f^{*}$ is continuous, $g^{*}$ is of class $\alpha$, and $(f, g)$ is the composition of

$$
T \stackrel{\left({ }^{1}, f\right)}{\rightarrow} \operatorname{Gr}(f) \stackrel{\left(f^{*}, g^{*}\right)}{\rightarrow} X \times Y .
$$

That completes the proof of the theorem.

For Borel maps of different classes we can deduce the following theorem. 
THEOREM 2. If in the assumptions of Theorem 1 the map $f$ is of class $\alpha$ and the map $g$ is of class $\beta$, then $(f, g)$ is of class $\min \{\alpha+\beta, \beta+\alpha\}$.

PROOF. The proof of Theorem 1 shows that $(f, g)$ is of class $\alpha+\beta$ and the map $(g, f)$ is of class $\beta+\alpha[\mathbf{K 1}, \S 31, \mathrm{III}]$. Since these maps differ only by a homeomorphism, it follows that $(f, g)$ is of class $\min \{\alpha+\beta, \beta+\alpha\}$.

Other variants of Theorem 1 can also be easily deduced. For example, since the Souslin subsets of a metric space contain the open sets, are closed to finite intersections and to unions of $\sigma$-locally finite subcollections, and since the composition of two Souslin measurable maps is again Souslin measurable, the above proof shows that $(f, g)$ will be Souslin measurable whenever $f$ and $g$ are. The corresponding statement for Borel measurable maps (without any reference to a class) would also follow if we knew that every Borel map between metric spaces had a class, but this remains an open problem in the nonseparable case (see [FrHJ]). Although the $\sigma$-algebra of sets having the Baire property possesses the above necessary closure properties, the composition of two maps with the Baire property may not have the Baire property, so the proof technique of Theorem 1 fails in this case. In fact, Kuratowski's problem of whether or not $(f, g)$ has the Baire property whenever $f$ and $g$ do $[\mathbf{K 2}, \S 8]$ evidently depends on additional axioms of set theory (see the discussion in [Fr, reference 18]). However, note that the above methods do show that $(f, g)$ will have the Baire property whenever $f$ has this property and $g$ is (say) Souslin measurable (cf. [K1, §32, III]).

Theorem 1 can also be used to deduce the following result on the Borel measurability of complex maps with countably many components.

THEOREM 3. Let $T, X_{1}, X_{2}, \ldots$ be nonempty metric spaces, and let $X$ denote the product spaces $\prod_{n=1}^{\infty} X_{n}$. Suppose $f: T \rightarrow X$ is such that each of the compositions $\pi_{n} \circ f$ is a Borel map of class $\alpha$, where $\pi_{n}$ is the nth projection map on $X$. Then $f$ will be a Borel map of class $\omega \alpha+1$.

PROOF. By Theorem 1 and finite induction, the map $f_{n}: T \rightarrow X$ whose first $n$ components are the same as the map $f$ and whose $k$ th component, for $k>n$, takes some constant value $x_{k} \in X_{k}$ (the same for each $n<k$ ) is a Borel map of class $n \alpha$. Since $f$ is clearly the pointwise limit of the functions $f_{n}$, it follows that $f$ is of class $\omega \alpha+1[\mathbf{K 1}, \S 31, \mathrm{VIII}]$.

REMARK. In the usual way, one can deduce from the above that finite and countable products of Borel maps of bounded class will again be Borel maps of bounded class (cf. [K1, §31, VI]).

2. Points of continuity of separately continuous maps. Suppose $T$ is a topological space, $Y$ is a metric space, and $f: T \rightarrow Y$ is a class-1 map (i.e., inverse images of open sets are $F_{\sigma}$ sets in $T$ ). If $Y$ is separable, then a classical theorem (essentially due to Baire) states that the set of points of continuity of $f$ is a comeagre set of $T$ (i.e., the complement of a set of first category in $T$ ) $[\mathbf{K} \mathbf{1}, \S 31$, $\mathrm{X}$ ]. For nonseparable $Y$ the following theorem gives a useful sufficient condition for a map to have this property (cf. [H1, Theorem 10] where it was assumed that $T$ was also metrizable). By a function base for a map $f: T \rightarrow Y$ we mean a collection $\mathscr{L}$ of subsets of $T$ such that, whenever $V \subset Y$ is an open set, $f^{-1}(V)$ is a union of sets from $\mathscr{L}$. 
THEOREM 4. Let $f: T \rightarrow Y$ be a class-1 map, where $T$ is any topological space and $Y$ is a metric space. If $f$ has a $\sigma$-locally finite function base $\mathscr{L}$, then $f$ is continuous at each point of some comeagre set of $T$.

ProOF. We first show that the sets in $\mathscr{L}$ can be modified to give a $\sigma$-locally finite function base of $F_{\sigma}$ sets in $T$. Let $\mathscr{V}$ be a $\sigma$-locally finite open base for $Y$. Since each member of $\mathscr{L}$ can be contained in $f^{-1}(V)$ for at most countably many $V \in \mathscr{V}$, the collection

$$
\mathscr{L}^{\prime}=\left\{\operatorname{cl}(L) \cap f^{-1}(V): L \subset f^{-1}(V), L \in \mathscr{L} \text { and } V \in \mathscr{V}\right\}
$$

will be a $\sigma$-locally finite family of $F_{\sigma}$ sets in $T$, which is easily shown to be a function base for $f$. We thus assume the members of $\mathscr{L}$ are $F_{\sigma}$ sets in $T$.

By the definition of a function base, it is clear that the set

$$
D=\bigcup\{L \backslash \operatorname{int}(L): L \in \mathscr{L}\}
$$

contains the set of discontinuity points of $f$. Since each $L \in \mathscr{L}$ is an $F_{\sigma}$ set in $T, L \backslash \operatorname{int}(L)$ will be of first category in $T$. Also, by the Banach Category Theorem $[\mathrm{K1}, \S 10, \mathrm{III}]$, the union of a locally finite collection of first category sets is again of first category in $T$, hence it follows that the set $D$ is of first category in $T$.

Fleissner $[\mathbf{F}]$ has shown that, assuming the existence of very large cardinals (supercompact), it is relatively consistent with the axioms of set theory for all Borel maps between metric spaces to have a $\sigma$-locally finite function base (cf. also $[\mathbf{F H J}])$. Thus, any class-1 map that arises in a natural way ought to have a function base of this type. (This principle is well illustrated in the proofs of the main results of [JR and HJT].)

Now let $T$ and $Y$ be as before, let $X$ be a second metric space, and suppose $f: X \times T \rightarrow Y$ is a separately continuous map (i.e., $f(\cdot, t)$ and $f(x, \cdot)$ are continuous for each fixed $t$ and $x$ ). Extending a result due to Kuratowski in the case when $T$ is also metric, we will first show that $f$ is a class-1 map (see also 3.1 below). Now this is precisely the sort of situation where we would expect to show that $f$ has a $\sigma$-locally finite function base. This is indeed the case as we will subsequently show in Theorem 6 below.

THEOREM 5. Let $f: X \times T \rightarrow Y$ be a separately continuous map, where $X$ and $Y$ are metric spaces. Then $f$ is of Borel class 1 .

PrOOF. Let $F$ be a given closed set in $Y$, and let $G_{n}$ be the open set of all points in $Y$ whose distance to $F$ is less than $1 / n$ for each $n \in \omega$. We need to show that $f^{-1}(F)$ is a $G_{\delta}$ set in $X \times T$.

For each $n \in \omega$, let $\left\{U_{n a}: a \in A_{n}\right\}$ be a cover of $X$ by nonempty open sets with diameters less than $1 / n$, and choose points $x_{n a} \in U_{n a}$ arbitrarily for each $a \in A_{n}$. We will show that

$$
f^{-1}(F)=\bigcap_{n \in \omega} \bigcup_{m>n} \bigcup_{a \in A_{m}} U_{m a} \times f_{x_{m a}}^{-1}\left(G_{n}\right) \quad\left(\text { where } f_{x}=f(x, \cdot)\right) .
$$

Suppose $(x, t) \in f^{-1}(F)$. Then, for each $n \in \omega$, we have $f(x, t) \in G_{n}$, so by the continuity of $f(\cdot, t)$ at $x$ there exist $m>n$ and $a \in A_{m}$ such that $x \in U_{m a}$ and $f\left(U_{m a} \times\{t\}\right) \subset G_{n}$; whence $(x, t) \in U_{m a} \times f_{x_{m a}}^{-1}\left(G_{n}\right)$. 
Conversely, suppose for each $n \in \omega$ there exist $m(n)>n$ and $a(n, m)$ in $A_{m(n)}$ such that $(x, t) \in U_{m(n) a(n, m)} \times f_{x(n)}^{-1}\left(G_{n}\right)$, where $x(n) \equiv x_{m(n) a(n, m)}$. Since both $x(n)$ and $x$ belong to the open sets $U_{m(n) a(n, m)}$ and the diameters of these sets tend to zero, we have $x(n) \rightarrow x$ as $n \rightarrow \infty$. Hence, by the continuity of $f(\cdot, t)$ we also have $f(x(n), t) \rightarrow f(x, t)$ as $n \rightarrow \infty$. Since $f(x(n), t) \in G_{n}$ for each $n \in \omega$, it follows that the distance from $f(x, t)$ to the closed set $F$ is zero, so $(x, t) \in f^{-1}(F)$ as required.

THEOREM 6. Let $f: X \times T \rightarrow Y$ be a separately continuous map, where $X$ and $Y$ are metric spaces. Then $f$ has a $\sigma$-locally finite function base (of closed sets). Consequently, $f$ is continuous at each point of some comeagre set in $X \times T$.

ProOF. For each $n \in \omega$ let $\left\{U_{n a}: a \in A_{n}\right\}$ be a locally finite open cover of $X$, consisting of nonempty sets having diameter less than $1 / n$. And let $\left\{V_{n b}: b \in\right.$ $\left.B_{n}, n \in \omega\right\}$, have exactly the same properties relative to the metric space $Y$. We choose, arbitrarily, points $x_{n a} \in U_{n a}$ and $y_{n b} \in V_{n b}$, for each $a \in A_{n}$ and $b \in B_{n}$, $n \in \omega$. For simplicity of notation, write

$$
W_{n a b}=U_{n a} \times f_{x_{n a}}^{-1}\left(V_{n b}\right) \quad\left(\text { where } f_{x_{n a}}=f\left(x_{n a}, \cdot\right)\right),
$$

and let us show that

$\mathscr{L}=\left\{W_{n a b} \cap f^{-1}\left(V_{m c}\right): n \in \omega, a \in A_{n}, b \in B_{n}, m \in \omega, c \in B_{m}\right.$, and $\left.y_{n b} \in V_{m c}\right\}$

is a $\sigma$-locally finite function base for $f$. Note that, for fixed $n,\left\{W_{n a b}: a \in A_{n}\right.$ and $\left.b \in B_{n}\right\}$ is a locally finite open cover of $X \times T$, and that each point $y_{n b}$, for fixed $b \in B_{n}$, can belong to only countably many sets in the $\sigma$-locally finite family $\left\{V_{c m}: c \in B_{m}\right.$ and $\left.m \in \omega\right\}$. Since $f$ is of class 1 by Theorem 5 , it follows that $\mathscr{L}$ is a $\sigma$-locally finite collection of $F_{\sigma}$ sets in $X \in T$. Note that any function with a $\sigma$-locally finite function base of $F_{\sigma}$ sets will have a similar function base of closed sets.

It remains to show that $\mathscr{L}$ is a function base for $f$. Let $(x, t)$ be any point in $X \times T$ and suppose $f(x, t)$ belongs to the open set $V$ in $Y$. We must find some $L \in \mathscr{L}$ satisfying $(x, t) \in L$ and $L \subset f^{-1}(V)$. Choose sequences $a(n)$ and $b(n)$ such that $(x, t) \in W_{n a(n) b(n)}$ for each $n \in \omega$. Then $x \in U_{n a(n)}$ and $f\left(x_{n a(n)}, t\right) \in V_{n b(n)}$. Since the diameters of $U_{n a(n)}$ tend to zero, the points $x_{n a(n)} \in U_{n a(n)}$ must converge to $x$, and so (by continuity) the sequence $f\left(x_{n a(n)}, t\right)$ converges to $f(x, t)$. Since also $y_{n b(n)} \in V_{n b(n)}$, and the diameters of these sets tend to zero, the sequence $y_{n b(n)}$ must also converge to $f(x, t)$. Now choose any $V_{m c}$ such that $f(x, t) \in V_{m c}$ and $V_{m c} \subset V$, and choose $n$ sufficiently large so that $y_{n b(n)} \in V_{m c}$. It then follows easily that $L=W_{n a(n) b(n)} \cap f^{-1}\left(V_{m c}\right)$ has the above desired properties.

3. Remarks and related results. The referee has supplied the following rather nice proof of Theorem 5 above: In the notation of Theorem 5 , first embed $Y$ in a Banach space $Z$. We may assume that the open covers of $X$ by the sets $U_{n a}$ are locally finite, that $\left\{g_{n a}: a \in A_{n}\right\}$ is a corresponding partition of unity, and that points $p_{n a} \in U_{n a}$ have been chosen for each $a \in A$ and $n \in \omega$. Now define $F_{n}: X \times T \rightarrow Z$ by

$$
F_{n}(x, t)=\sum\left\{g_{n a}(x) f\left(p_{n a}, t\right): a \in A_{n}\right\} .
$$


Then $F_{n}$ is continuous and converges to $f$ pointwise as $n \rightarrow \infty$; whence $f$ is of class 1. Note that it also follows from this that $f$ is continuous at each point of some comeagre set in $X \times T$ by a result of Fort [Fo, Theorem 2]. This representation of $f$ as a pointwise limit of continuous functions can also be used to give an alternate proof that $f$ will have a $\sigma$-locally finite function base using methods similar to those in the proof of Lemma 3.2 of [H3].

We can give a general version of Theorem 1 for maps from a measurable space to arbitrary topological spaces by utilizing the concept of a hereditarily-additive function base introduced in [H3]. Let $\mathscr{M}$ be a collection of subsets of a set $T$ containing the empty set and closed to finite intersections. A collection $\left\{M_{a}: a \in\right.$ $A\} \subset \mathscr{M}$ is said to be $\mathscr{M}$-hereditarily additive if, whenever $\left\{N_{a}: a \in A\right\} \subset \mathscr{M}$, then $\bigcup_{a \in A} N_{a} \cap M_{a}$ belongs to $\mathscr{M}$. If $X$ is a topological space, then $f: T \rightarrow X$ is said to be $\mathscr{M}$-measurable if, whenever $U \subset X$ is open, then $f^{-1}(U) \in \mathscr{M}$.

THEOREM 7. Let $T$ and $\mathscr{M}$ be as above, and suppose $f: T \rightarrow X$ is $\mathscr{M}$ measurable and $g: T \rightarrow Y$ has an $\mathscr{M}$-hereditarily additive function base. Then the complex map $(f, g): T \rightarrow X \times Y$ is $\mathscr{M}$-measurable.

ProOF. Let $\mathscr{B} \subset \mathscr{M}$ be an $\mathscr{M}$-hereditarily additive function base for $g$. Let $W \subset X \times Y$ be an open set and write $W=\bigcup\left\{U_{a} \times V_{a}: a \in A\right\}$ for open setś $U_{a} \subset X$ and $V_{a} \subset Y$. For each $B \in \mathscr{B}$ define

$$
M_{B}=f^{-1}\left[\bigcup\left\{U_{a}: a \in A \text { and } B \subset g^{-1}\left(V_{a}\right)\right\}\right],
$$

and let us show that $(f, g)^{-1}(W)=\bigcup_{B \in \mathscr{B}} M_{B} \cap B$. Since $\mathscr{B}$ is $\mathscr{M}$-hereditarily additive and $M_{B} \in \mathscr{M}$ for each $B \in \mathscr{B}$, it will follow that $(f, g)$ is $\mathscr{M}$-measurable.

If $t \in(f, g)^{-1}(W)$, then we have $t \in f^{-1}\left(U_{a}\right) \cap g^{-1}\left(V_{a}\right)$ for some $a \in A$. Thus, by the definition of a function base, for some $B \in \mathscr{B}$ we must have $t \in B \subset g^{-1}\left(V_{a}\right)$; whence $t \in M_{B} \cap B$.

Conversely, if $t \in M_{B} \cap B$ for some $B \in \mathscr{B}$, then for some $a \in A$ we have $t \in$ $f^{-1}\left(U_{a}\right)$ and $B \subset g^{-1}\left(V_{a}\right)$; thus $(f(t), g(t)) \in U_{a} \times V_{a}$ and so $t \in(f, g)^{-1}(W)$.

REMARKS. The following are examples of when an $\mathscr{M}$-measurable map $f: T \rightarrow$ $X$ has an $\mathscr{M}$-hereditarily additive function base, where $X$ is a metric space and $T$ and $\mathscr{M}$ are as before.

(1) $T$ is an absolutely analytic metric space and $\mathscr{M}$ is either the collection of sets of additive class $\alpha$ in $T$ or the collection of Souslin-F sets of $T$ [H1].

(2) Same as (1) except $T$ is any metric space and Fleissner's axiom (P) holds [F].

(3) $\mathscr{M}$ is a $\sigma$-algebra and $f$ is extended Bochner measurable relative to $\mathscr{M}$ (i.e., $f$ is a pointwise limit of a sequence of functions $f_{n}: T \rightarrow X$ such that $\left\{f_{n}^{-1}(x): x \in X\right\}$ is $\mathscr{M}$-hereditarily additive for each $n \in \omega)$ [H3, Theorem 3.3].

\section{REFERENCES}

[E] R. Engelking, On Borel sets and B-measurable functions in metric spaces, Ann. Soc. Math. Polon. (Comm. Math.) 10 (1967), 145-149.

[F] W. G. Fleissner, An axiom for nonseparable Borel theory, Trans. Amer. Math. Soc. 251 (1979), 309-328.

[FHJ] W. G. Fleissner, R. W. Hansell and H. J. K. Junnila, PMEA implies Proposition P, Topology Appl. 13 (1982), 255-262. 
[Fo] M. K. Fort, Jr., Category theorems, Fund. Math. 42 (1955), 276-288.

[Fr] D. H. Fremlin, Measure-additive coverings and measurable selectors (to appear).

[FrHJ] D. H. Fremlin, R. W. Hansell and H. J. K. Junnila, Borel functions of bounded class, Trans. Amer. Math. Soc. 277 (1983), 835-849.

[H1] R. W. Hansell, Borel measurable mappings for nonseparable metric spaces, Trans. Amer. Math. Soc. 161 (1971), 145-169.

[H2] _ Hereditarily-additive families in descriptive set theory and Borel measurable multimaps, Trans. Amer. Math. Soc. 278 (1983), 725-749.

[H3] - Extended Bochner measurable selectors, Math. Ann. 277 (1987), 79-94.

[HJT] R. W. Hansell, J. E. Jayne and M. Talagrand, First class selectors for weakly upper semicontinuous multi-valued maps in Banach spaces, J. Reine Angew. Math. 361 (1985), 201-220.

[JR] J. E. Jayne and C. A. Rogers, Borel selectors for upper semicontinuous set-valued maps, Acta Math. 155 (1985), 41-79.

[K1] K. Kuratowski, Topology, Vol. 1, Academic Press, New York, PWN, Warsaw, 1966.

[K2] _ Quelques problèmes concernant espaces métriques nonséparables, Fund. Math. 25 (1935), 532-545.

[M] D. Montgomery, Non-separable metric spaces, Fund. Math. 25 (1935), 527-533.

[S] A. H. Stone, Some problems of measurability (Topology Conf., Blacksburg, Virginia, 1973), Lecture Notes in Math., vol. 375, Springer-Verlag, Berlin and New York, 1974, pp. 242-248.

Department of Mathematics, University of ConneCticut, Storrs, ConneCtICUT 06268 